\title{
Eficacia clínica de la biopsia prostática. Experiencia en nuestro centro 1990-2002
}

\author{
Escudero Bregante JF, López Cubillana P, Cao Avellaneda E, López López AI, Maluff Torres A, \\ López González PA, Prieto González A, Rigabert Montiel M, Tornero Ruíz JI, Gómez Gómez GA, \\ Pérez Albacete M.
}

Servicio de Urología del Hospital Universitario Virgen de la Arrixaca. Murcia.

Actas Urol Esp. 2008;32(7):713-716

\section{RESUMEN}

EFICACIA CLÍNICA DE LA BIOPSIA PROSTÁTICA. EXPERIENCIA EN NUESTRO CENTRO 1990-2002

Introducción: La biopsia prostática es la única herramienta válida para diagnosticar la existencia de cáncer de próstata. Las indicaciones para su realización según la Asociación Europea de Urología son la existencia de PSA elevado, velocidad aumentada y la evidencia de un tacto rectal sospechoso.

Objetivos: Valoración tanto de la utilidad clínica de la biopsia prostática, como del PSA como marcador de cáncer de próstata en nuestro medio y la indicación y eficacia de sucesivas biopsias.

Material y Métodos: Efectuamos una revisión manual de las biopsias realizadas en nuestro servicio entre los años 1990 y 2002, valorando nivel de PSA previo a la biopsia, número de cilindros obtenidos y datos de la biopsia. Se llevó a cabo un estudio estadístico descriptivo mediante el programa estadístico SPSS 12.0

Resultados: Se han realizado 1202 biopsias en este periodo, con un porcentaje de positividad global del 36.96\%. El PSA previo a la biopsia (disponible en las biopsias realizadas entre los años 1999 y 2002: 578 biopsias, $48.08 \%$ del total) era $>10 \mathrm{ng} / \mathrm{ml}$ en el $55,88 \%$ de estos pacientes, $4-10 \mathrm{ng} / \mathrm{ml}$ en el $39.27 \%$ y 0-4ng/ml en el 4,84\%. La media y mediana de PSA es de 19,09 (error típico: 1,87) y 10,6, respectivamente. La positividad de la biopsia aumenta con el nivel de PSA: $21,4 \%$ en pacientes con PSA<4; $25,11 \%$ con PSA $4-10$ y $48,61 \%$ con PSA > 10. Fue realizada rebiopsia prostática en 132 pacientes (21,97\% positivas) El 88,36\% de los cánceres fueron diagnosticados en la primera biopsia, y un 6,62\% en la segunda $(94,98 \%$ de los diagnósticos de cáncer de próstata se realizaron con las 2 primeras biopsias). Conclusiones: Los datos obtenidos en el estudio mediante el análisis descriptivo de nuestra serie se encuentran en concordancia con lo publicado en otros estudios y publicaciones. Existe una necesidad de aumentar la rentabilidad diagnóstica de la biopsia de próstata, para lo cual hemos introducido un protocolo de biopsia bajo anestesia local con el fin de poder aumentar el número de cilindros obtenidos.

Palabras clave: Cáncer de próstata. Biopsia prostática. Diagnóstico. Eficiencia.

\section{ABSTRACT}

CLINICAL EFFICACY OF PROSTATIC BIOPSY. EXPERIENCE IN OUR CENTER FROM 1990 TO 2002

Introduction: The prostate biopsy is the only valid tool to diagnose the existence of cancer of prostate. The indications of the biopsy, according with EAU, are the existence of high PSA, increased velocity PSA and a rectal suspicious tact.

Objectives: validation of the utility of the prostate biopsy, to know the value of the PSA as a marker of prostate cancer in our way and to value the indication and efficiency of repeated biopsies.

Material and methods: we practice a manual review of the biopsies in our hospital, between the years 1990 and 2002 . We study the level of PSA before the biopsy, number of prostatic cores and histologic information of the biopsy. A statistical descriptive and inferencial study has been performed by SPSS 12.0 package.

Results: The total number of biopsies registered was a 1202, with $36.96 \%$ of biopsy positive. The PSA before the biopsy (available in the biopsies realized between the year 1999 and 2002: 578 biopsies, $48.08 \%$ of the whole) was $>10 \mathrm{ng} / \mathrm{ml}$ in $55,88 \%$ of these patients, $4-10 \mathrm{ng} / \mathrm{ml}$ in $39.27 \%$ and $0-4 \mathrm{ng} / \mathrm{ml}$ in $4.84 \%$. The average and PSA's median is of 19.09 (standard error: 1.87) and 10.6, respectively. The positividad of the biopsy increases with PSA's level: 48,61 \% with PSA> 10; $25.11 \%$ with PSA 4-10 and $21,4 \%$ in patients with PSA $<4$. There was realized prostate rebiopsy ( 2 ó more biopsies) in 132 patients $(21,97 \%$ positive) $88,36 \%$ of the cancers was diagnosed in the first biopsy, and $6.62 \%$ in the second one $194,98 \%$ of the diagnoses of cancer of prostate carried out with the first 2 biopsies).

Conclusions: The information obtained in the study by means of the descriptive analysis of our series meets in conformity the published in other studies and publications. There exists a need to increase the diagnostic profitability of the biopsy of prostate, for which we have introduced a protocol of biopsy under local anesthesia in order to be able to increase the number of obtained cylinders. Keywords: Prostate cancer. Prostate biopsy. Diagnosis. Efficacy. 
$\mathrm{L}^{\mathrm{a}}$ a biopsia prostática es la única herramienta válida para diagnosticar la existencia de cáncer de próstata ${ }^{1}$. Las indicaciones para su realización según la Asociación Europea de Urología son PSA patológico, velocidad de PSA elevada y tacto rectal sospechoso ${ }^{1}$.

Para valorar la eficacia diagnóstica de la biopsia prostática en nuestro servicio hemos realizado un estudio retrospectivo de todas las biopsias realizadas en nuestro servicio entre 1990 y 2002.

\section{OBJETIVOS}

a) Primario: valoración de la utilidad clínica de la biopsia prostática.

b) Secundarios:

b.1. conocer el valor del PSA como marcador de cáncer de próstata en nuestro medio.

b.2. valorar la indicación y eficacia de sucesivas biopsias.

\section{MATERIAL Y MÉTODOS}

Realizamos una revisión manual de las biopsias registradas en el Servicio de Anatomía Patológica entre los años 1990 y 2002, y se recogieron datos de filiación del paciente (nombre, edad, número de historia, etc.), datos de laboratorio (PSA, Ratio PSA $\mathrm{L} / \mathrm{T}$ ), datos de la biopsia (tamaño prostático, presencia de nódulos) y datos del estudio histológico de las muestras (Gleason, lateralidad del tumor, extensión del mismo, etc).

La biopsia prostática se realizó en todos los casos vía transrectal, con control ecográfico (desde el año 1996). La preparación para la biopsia incluyó enema de limpieza y profilaxis antibiótica con Ciprofloxacino $500 \mathrm{mg}$ vía oral cada 12 horas durante 5 días, con inicio de la misma 1 hora antes de la biopsia. La muestra prostática se obtuvo con una pistola de biopsia marca VITESSE de la casa Optimed, obteniendo cilindros de 18G de diámetro y $2 \mathrm{~cm}$ de longitud. Las biopsias fueron realizadas por 8 médicos diferentes.

Con todos estos datos se confeccionó un registro con 1202 biopsias, sobre el cuál se realizó estudio estadístico descriptivo mediante el programa estadístico SPSS 12.0. Las variables cuantitativas se expresaron como media \pm desviación estándar (DE). Las variables cualitativas se expresaron como frecuencias y porcentajes.

\section{RESULTADOS}

El número total de biopsias registrado fue 1202 . Hemos observado un incremento continuo en su realización, desde las 28 en 1991 hasta las $187 \mathrm{del}$ 2002. Se expone gráficamente en la Figura 1.

El porcentaje global de biopsias positivas en nuestra serie es del $36.96 \%$. Las distintas variaciones observadas anualmente se exponen a continuación de manera gráfica (Tabla 1).

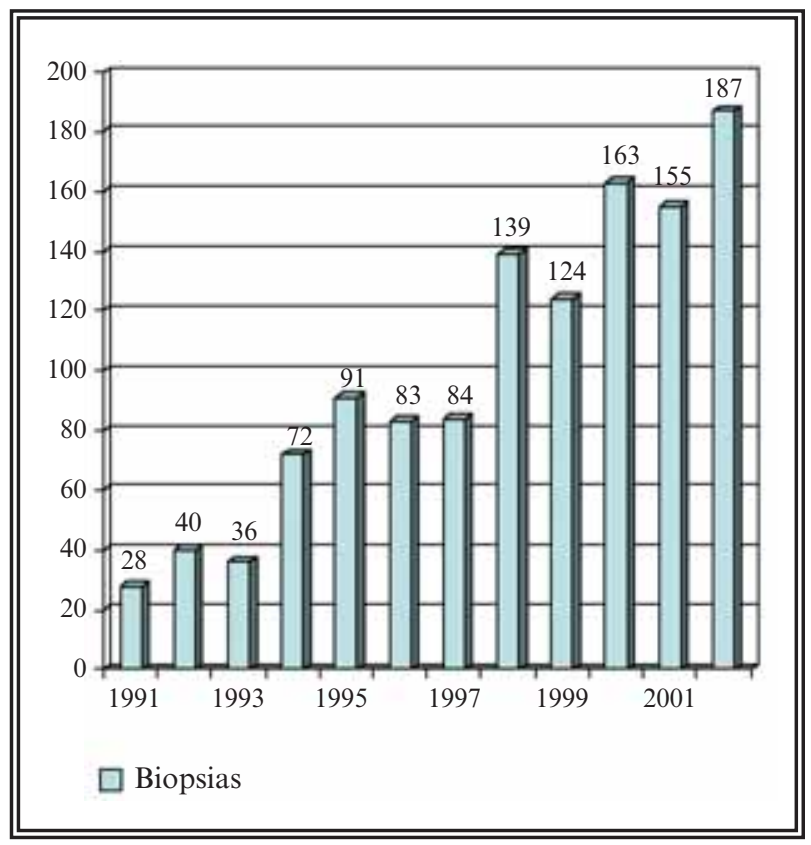

FIGURA 1. Evolución histórica de nuestras biopsias.

Tabla 1. Porcentaje de positividad de la biopsia según año

\begin{tabular}{lccc}
\hline Año & Biopsias & $\begin{array}{c}\text { Biopsias } \\
\text { positivas }\end{array}$ & $\begin{array}{c}\text { \% de } \\
\text { positividad }\end{array}$ \\
\hline 1991 & 28 & 18 & 64,29 \\
1992 & 40 & 20 & 50,00 \\
1993 & 36 & 22 & 61,11 \\
1994 & 72 & 25 & 34,72 \\
1995 & 91 & 29 & 31,87 \\
1996 & 83 & 19 & 22,89 \\
1997 & 84 & 36 & 42,86 \\
1998 & 139 & 36 & 25,90 \\
1999 & 124 & 45 & 36,29 \\
2000 & 163 & 71 & 43,56 \\
2001 & 155 & 59 & 38,06 \\
2002 & 187 & 68 & 36,36 \\
\hline
\end{tabular}


El PSA previo a la biopsia está disponible en las biopsias realizadas entre los años 1999 y 2002 (578 biopsias, 48,08\% del total). El nivel de PSA en el momento de la biopsia era $>10 \mathrm{ng} / \mathrm{ml}$ en el $55,88 \%$ de estos pacientes, 4-10 ng/ml en el 39,27\% y 0$4 \mathrm{ng} / \mathrm{ml}$ en el $4,84 \%$. La media y mediana de PSA es de 19,09 (error típico: 1,87) y 10,6, respectivamente (Tablas 2 y 3).

La positividad de la biopsia prostática varió en función del PSA previo a la misma, así para los pacientes con un PSA> 10 el 48,61\% de las biopsias realizadas a eran positivas, frente a un $25,11 \%$ para los pacientes con PSA entre 4 y 10 , y un $21,4 \%$ en pacientes con PSA $<4$ (Tabla 4 ).

Por último, evaluamos las rebiopsias realizadas. Encontramos 132 pacientes sometidos a 2 ó más biopsias, de los cuales 29 pacientes (un 21,97\% de los mismos) fueron diagnosticados de cáncer de próstata. El 88,36\% de los cánceres se diagnostican en la primera biopsia, en la segunda biopsia se diagnostican un 6,62\% (total acumulado: 94,98\%) (Figura 2 y Tabla 5).

Tabla 2. Número de biopsias en función del PSA y el porcentaje que ello representa respecto al total de la muestra

\begin{tabular}{lcc}
\hline Biopsias & $\mathbf{N}^{\mathbf{0}}$ pacientes & \% representado \\
\hline PSA $<4$ & 28 & $4,84 \%$ \\
PSA $4-10$ & 227 & $39,27 \%$ \\
PSA $>10$ & 323 & $55,88 \%$ \\
\hline
\end{tabular}

Tabla 3. Estadística descriptiva del PSA

\begin{tabular}{lr}
\hline \multicolumn{2}{c}{ Estadísticos } \\
\hline $\mathrm{N}$ Válidos & 551 \\
$\quad$ Perdidos & 27 \\
Media & 19,09 \\
Error tip. de la media & 1,875 \\
Mediana & 10,60 \\
Mínimo &, 01 \\
Máximo & 932,90 \\
\hline
\end{tabular}

Tabla 4. En la misma se muestra la positividad de la biopsia en función del valor de PSA

\begin{tabular}{lll}
\hline PSA $<4$ & $6 / 28$ & $21,4 \%$ \\
PSA $4-10$ & $57 / 227$ & $25,11 \%$ \\
PSA $>10$ & $157 / 323$ & $48,61 \%$ \\
\hline
\end{tabular}

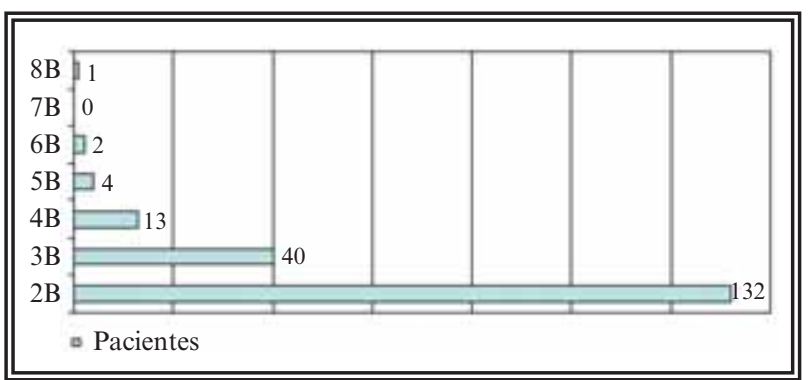

FIGURA 2. En la tabla se muestran los pacientes sometidos a rebiopsia.

Tabla 5. Positividad de la biopsia según número de biopsias y porcentaje sobre el total

\begin{tabular}{lcccc}
\hline & $\mathbf{n}^{\mathbf{0}}$ & $\mathbf{( + )}$ & $\mathbf{\%}$ & \% sobre el total \\
\hline Primera & 996 & 387 & $38,86 \%$ & $88,36 \%$ \\
Segunda & 132 & 29 & $21,97 \%$ & $6,62 \%$ \\
Tercera & 40 & 16 & $40 \%$ & $3,65 \%$ \\
Cuarta & 13 & 4 & $30,7 \%$ & $0,91 \%$ \\
Quinta & 4 & 2 & $50 \%$ & $0,45 \%$ \\
\hline
\end{tabular}

\section{DISCUSION}

En el momento actual el PSA y el tacto rectal continúan siendo los principales marcadores e indicadores para la realización de la biopsia de próstata, único recurso disponible en el diagnóstico de certeza del cáncer de próstata.

En nuestra serie la indicación de biopsia de próstata se ha realizado en función del PSA total, del PSA L/T y del tacto rectal:

1. Tacto rectal patológico.

2. Pacientes con PSA 4 - $10 \mathrm{ng} / \mathrm{ml}$ y PSA ratio < 0,20

3. PSA $>10$ con independencia del valor del PSA ratio.

Existe una tendencia hacia la reducción de los límites del PSA para indicar la biopsia. Autores como F. Schröder. en el Estudio Randomizado Europeo de Screening del Cáncer de Próstata (ERSPC), en la rama de Países Bajos propusieron indicar la biopsia a todo paciente con un PSA mayor de $3 \mathrm{ng} / \mathrm{ml}^{2}$, otros autores hablan de límites aun más bajos de PSA para indicar la biopsia, como el grupo de Catalona que sugiere biopsia a todo paciente con PSA $>2,5 \mathrm{ng} / \mathrm{ml}^{3}$.

Asímismo, nuevos marcadores adquieren mayor protagonismo en el diagnóstico del cáncer de próstata, como es la velocidad de PSA, entendida como incremento absoluto temporal de sus valores. Autores como Smith y Fang describen que velocidades de PSA $>0,75 \mathrm{ng} / \mathrm{ml} /$ año y $0,5 \mathrm{ng} / \mathrm{ml} /$ año se asocian con aumento del riesgo relativo de cáncer de prós- 
tata de 7,2 y 6,53 respectivamente r,5 $^{4,}$ Otros autores como Roobol no han podido confirmar la utilidad de este marcador 6 .

La utilidad clínica global en nuestra serie se cifra en el $36,96 \%$, porcentaje concordantes con los obtenidos en las diferentes series revisadas. Otras series, como la de Schröder, lo cifra en el 29,2\%. Esta diferencia puede ser debida al alto porcentaje de pacientes que registramos con PSA de 10 o superior en el momento de la biopsia. Si analizamos los resultados en función del intervalo de PSA vemos cómo en la franja de pacientes con $\mathrm{PSA}<4 \mathrm{ng} / \mathrm{ml}$ obtuvimos una positividad del 21,4\% (frente a una positividad de $21,8 \%$ en la serie de Schröder), en los pacientes con PSA de $4-10 \mathrm{ng} / \mathrm{ml}$ una positividad del $25,11 \%$ (frente a un $27,9 \%$ ) y en pacientes con PSA $>10 \mathrm{ng} / \mathrm{ml}$ la positividad fue de $48,61 \%$, frente al $57,6 \%$ de la serie comparada ${ }^{7}$.

La indicación de rebiopsia más frecuente e importante fue un PSA anormalmente elevado de forma persistente. En nuestra serie, de los 129 pacientes sometidos a una rebiopsia fue positiva en 29 de ellos (21,97\%), un $6,62 \%$ del total de positividades. En la segunda rebiopsia obtuvimos un $40 \%$ de positividad (3,65\% del total) y con la tercera un 30,7\% (0,91\% del total). En los estudios revisados, se muestra una tendencia a la pérdida de rentabilidad conforme se aumenta el número de rebiopsia, tal y como explican autores como Djavan, que presenta un $10 \%$ en la segunda biopsia, un 5\% en la tercera y un $4 \%$ en la cuarta biopsia $^{8}$. Según éstos, las dos primeras biopsias están justificadas, pero, a partir de la tercera, se debe evaluar al paciente en función de su riesgo concreto y de forma individualizada, por lo que no recomiendan de forma sistemática la tercera y sucesivas biopsias ${ }^{8}$.

En lo que si parece haber un acuerdo es en la necesidad de aumentar el número de cilindros de la biopsia en base al volumen, PSA y edad del paciente (como el nomograma de Viena propuesto por Djavan), realizando la biopsia al paciente bajo anestesia local y con cobertura antibiótica. Esta tendencia es sostenida por otros estudios como los de Gore JL y Chang JJ en los que se habla de un aumento en la detección de cánceres de próstata de un $24-36 \%$ por el hecho de aumentar de 6 cilindros a 10-129,10.

En cuanto al número de cilindros que se deben obtener no existe un acuerdo unánime. En nuestro servicio hemos implantado la biopsia de 5 cilindros por lóbulo más biopsia individualizada de nódulos tanto clínicos como ecográficos. Los resultados a los dos años serán comunicados en breve.
Algo similar ocurre en cuanto a la administración del anestésico. En nuestro medio decidimos realizar el gesto anestésico mediante la punción del espacio existente entre la vesícula seminal y la próstata (zona conocida ecográficamente como "signo del monte Everest”). Existen otros protocolos de anestesia en distintas localizaciones de la próstata, como el propuesto por Costello ${ }^{11}$.

\section{CONCLUSIONES}

La rentabilidad global de nuestra serie es de un $36,96 \%$. En función del PSA, en pacientes con PSA< $4 \mathrm{ng} / \mathrm{ml}$ la rentabilidad se sitúa en el $21,4 \%$, en pacientes con PSA entre 4 y 10 la rentabilidad es de un $25,11 \%$ y en pacientes con PSA > $10 \mathrm{ng} / \mathrm{ml}$ la rentabilidad es de un 48,61\%. Los datos obtenidos en el estudio mediante el análisis descriptivo de nuestra serie se encuentran en concordancia con lo publicado en otros estudios y publicaciones.

\section{REFERENCIAS}

1. Catalona WJ, Smith DS, Ratliff TL, Dodds KM, Coplen DE, Yuan JJ, et al. Measurement of prostate-specific antigen in serum as a screening test for prostate cancer. N Engl J Med. 1991;324(17):11561161.

2. Schröder et al. Prostat specific antigen based early detection of prostate cancer validation of screening without rectal examination. Urology. 2001;57(1):83-90.

3. Catalona WJ, Ramos CG, Carvalhal GF, Yan Y. A cutoffs to enhance detection of curable prostate cancer. Urology. 2000;55(6):791-795.

4. Smith DS, Catalona WJ. Rate of change in serum prostate specific antigen levels as a method for prostate cancer detection. J Urol. 1994;152(4): 1163-1167.

5. Fang J, Metter EJ, Landis P, Carter HB. PSA velocity for assessing prostate cancer risk in mem with PSA levels between 2.0 and 4.0 ng/ml. Urology. 2002;59(6):889-893.

6. Roobol MJ et al. A comparison of first and repeat (4 years later) prostate cancer screening in a randomised cohort of a-symptomatic men aged 55 - 76 years using a biopsy indication of $3.0 \mathrm{ng} / \mathrm{ml}$ (results of ERSPC - Rotterdam). Prostate 2005.

7. Raaijmakers $\mathrm{R}$ et al. Prostate-specific antigen change in the European Randomized Study of Screening for Prostate Cancer, section Rotterdam. Urology 2004 febrero; 63 (2): 316-20.

8. Djavan B, Milani S, Remzi M. Prostate biopsy: who, how and when. An update. Can J Urol. 2005; 12(1):44-48.

9. Gore JL, Shariat SF, Miles BJ, Kadmon D, Jiang N, Wheeler TM, et al. Optimal combinations of systematic sextant and laterally directed biopsies for the detection of prostate cancer. J Urol. 2001;165(5): 1554-1559.

10. Chang JJ, Shinohara K, Bhargava V, Presti JC Jr. Prospective evaluation of lateral biopsies of the peripheral zone for prostate cancer detection. J Urol. 1998;160(6 Pt 1):2111-2114.

11. Costello TG, Costello AJ. Anaesthesia for laser prostatectomy. Anaesth Intensive Care. 1994;22(4):454-457.

Correspondencia autor: Dr. J.F. Escudero Bregante

Servicio de Urología. Hospital Universitario Virgen de la Arrixaca

Ctra. Madrid-Cartagena, s/n - 30120 El Palmar (Murcia).

Tel.: 968369500

E-mail autor: jfeb13@hotmail.com

Información artículo: Original - Cáncer de próstata

Trabajo recibido: diciembre 2007

Trabajo aceptado: abril 2008 